\title{
HIPOMINERALIZACIÓN INCISIVO MOLAR Y FACTORES ETIOLÓGICOS AMBIENTALES. REVISIÓN DE LA LITERATURA
}

\author{
MOLAR INCISOR HYPOMINERALIZATION AND ETIOLOGICAL ENVIRONMENTAL \\ FACTORS. REVIEW OF THE LITERATURE \\ María Solís-Espinoza \\ mese111@hotmail.com \\ ORCID: 0000-0003-3724-2946 \\ Catherine Sharlot Alarcón-Calle ${ }^{1}$ \\ sharlot2989@gmail.com \\ ORCID: 0000-0003-4665-191X
}

\section{RESUMEN}

La etiología de la hipomineralización incisivo-molar (HIM) aún no está totalmente definida, por lo que el objetivo del presente artículo fue hacer una revisión de la literatura de los posibles factores etiológicos asociados. Se realizó una búsqueda inicial en las bases de datos Elsevier, EBSCO, PubMed y Medline usando las siguientes palabras clave: molar incisor hypomineralisation, hypoplasia, dental enamel defects epidemiology, etiology. Se incluyeron artículos en inglés y español, estudios epidemiológicos de casos y controles de HIM, descripción del método de diagnóstico y registro, y descripción del factor asociado, publicados entre 1987 y 2018. Tomando en consideración 40 artículos que cumplían con los criterios de búsqueda. Se dividieron los factores etiológicos encontrados en tres etapas: prenatal, perinatal y posnatal. La causa exacta aún no está del todo definida, pero con base en los estudios tenemos indicios que nos podrían ayudar a prevenir este tipo de defectos. Sin embargo, son necesarios

Palabras clave: Hipomineralización incisivo-molar, hipoplasia, defectos esmalte dental, epidemiología, etiología

\section{ABSTRACT}

The etiology of molar incisor hypomineralization $(\mathrm{MIH})$ is not yet fully defined, therefore the objective of this article was to review the literature on the possible associated etiological factors. An initial search was performed in the Elsevier, EBSCO, PubMed and Medline databases using the following keywords: molar incisor hypomineralization, hypoplasia, dental enamel defects epidemiology, and etiology. Articles in English and Spanish were included as well as epidemiological studies of cases and controls

Citar como: Solís-Espinoza ME, Alarcón-Calle CS. Hipomineralización incisivo molar y factores etiológicos ambientales: Revisión de la literatura. Rev Cient Odontol (Lima). 2019;7(1): 140-147.

1 División de Odontopediatría, Carrera de Estomatología, Universidad Cientifica del Sur. Lima, Perú. 
of $\mathrm{MIH}$, descriptions of the method of diagnosis and registration, and descriptions of associated factors published between 1987 and 2018. Forty articles met the search criteria. The etiological factors found were divided into three stages: prenatal, perinatal and postnatal. While the cause of $\mathrm{MIH}$ has yet to be fully defined, the studies available describe indications to help prevent this type of defects. Nonetheless, more longitudinal studies are necessary to determine the exact etiology of this alteration, and pediatricians and pediatric dentists should take into account the importance of early, adequate management of $\mathrm{MIH}$

Keywords: molar incisor hypomineralization, hypoplasia, dental enamel defects epidemiology, etiology.

\section{INTRODUCCIÓN}

La hipomineralización dental fue descrita por primera vez por Koch y cols. en 1987, con el término hipomineralización idiopática para referirse a un defecto cualitativo en el esmalte. Weerheinjn, en 2001, modificó el término a hipomineralización incisivo-molar (HIM) cuando se presentaban defectos en los primeros molares e incisivos permanentes $\left({ }^{1-4}\right)$. Estos cambios cualitativos, que son parte de los defectos de desarrollo del esmalte (DDE), son perceptibles porque el esmalte presenta opacidades demarcadas que varían en color del blanco al amarillo/marrón, con una mayor o menor nitidez en la demarcación entre el esmalte afectado y el normal ${ }^{(5-7)}$.

La amelogénesis de los primeros dientes permanentes se da entre las 28 semanas en el útero y el primer año de vida. La formación de esmalte se ha dividido en tres etapas principales. La primera es la etapa secretora, en la que los ameloblastos producen grandes cantidades de matriz del esmalte, dentro de las cuales se forman delgadas cintas de esmalte, principalmente hidroxiapatita. Casi inmediatamente después de depositarse los cristales, crecen principalmente en longitud y la capa de esmalte aumenta de espesor. La etapa de maduración comienza cuando se completa el espesor del esmalte y los ameloblastos secretores se transforman, a través de una etapa de transición, en ameloblastos maduros, los cuales son responsables de la degradación de la matriz del esmalte. Por último, está la etapa de mineralización, en la que el esmalte se endurece a medida que los cristales crecen, dando como resultado un tejido que contiene más del $95 \%$ de materia inorgánica $\left({ }^{4}\right)$.

La etiología exacta aún no está totalmente definida; sin embargo, hay indicios que podrían ser mecanismos fisiopatológicos de varias enfermedades o condiciones que afectarían a la madre y al bebé en las etapas pre, peri o posnatal ${ }^{(1-4)}$. Según el momento y la duración de estos factores, los dientes pueden sufrir diversas condiciones patológicas. Cuando los defectos se producen en la etapa secretora, el resultado es generalmente hipoplasia del esmalte, mientras que las alteraciones en la etapa de maduración llevan a una hipomineralización del esmalte $\left(^{8-10}\right)$.

Estas lesiones, de acuerdo con su severidad, podrían causar sufrimiento en los pacientes, ya que causan sensibilidad y mayor riesgo de caries en las piezas afectadas, lo que afecta la calidad de vida. $\left({ }^{11-13}\right)$ Por lo tanto es un desafío para el odontólogo, ya que las piezas pasan por constantes tratamientos $y$, muchas veces, el mal manejo del dolor con anestésicos locales puede generar una conducta negativa del paciente en las citas dentales. $\left({ }^{14}\right)$ 
El objetivo del presente artículo es hacer una revisión de la literatura sobre los posibles factores etiológicos asociados a la hipomineralización incisivo-molar.

\section{METOdOLOGíA}

Se realizó la búsqueda en las siguientes bases de datos: Elsevier, EBSCO, PubMed y Medline, a partir de una combinación de los siguientes temas médicos: molar incisor hypomineralisation, hypoplasia, dental enamel defects epidemiology, etiology. Se incluyeron los artículos en inglés y español, estudios epidemiológicos de casos y controles de HIM, descripción del método de diagnóstico y registro, descripción de un factor asociado, publicados entre 1987 y 2018. Se realizó una tabla a modo de resumen de los factores prenatales, perinatales y posnatales revisados.

Tabla 1. Posibles factores etiológicos del him

\begin{tabular}{|c|c|c|}
\hline \multicolumn{3}{|c|}{ Factores asociados con la etiología de la HIM } \\
\hline Periodo prenatal & Periodos perinatales & Periodo posnatal \\
\hline Tabaco y alcohol & Hipoxia & Procesos febriles \\
\hline \multirow[t]{5}{*}{ Ecografías } & Parto prematuro & Enfermedades como otitis \\
\hline & Bajo peso al nacer & Media aguda, neumonía, asma \\
\hline & & Antibióticos (amoxicilina) \\
\hline & & Uso de paracetamol e ibuprofeno \\
\hline & & Hipomineralización del molar deciduo \\
\hline
\end{tabular}

\section{PRENATALES}

Alcohol: Jimenez et al. investigaron con animales demostrando que el etanol (alcohol) puede provocar cambios en la diferenciación celular y la mineralización del esmalte $\left({ }^{15}\right)$, esta relación la comprobó Elfrick et al. en su estudio de $2104\left({ }^{16}\right)$.

Ecografías: En el estudio de Ghanim y Chaimay hallaron relación con las exposiciones múltiples a las ondas de ultrasonido en el último trimestre; sin embargo, no queda muy claro si es por la duración, la intensidad o la frecuencia de exposición a estas ondas que se genera un efecto negativo en el desarrollo del feto. Por otro lado, podría ser que, por causa de otras enfermedades, existió la necesidad de frecuente exposición a las ondas de ultrasonido. Si este es el caso, la enfermedad podría ser el potencial factor causal en lugar de la ecografía. $\left({ }^{17,18}\right)$.

\section{PERINATALES}

Parto prematuro: Estudios recientes han encontrado que el bajo peso al nacer o el nacimiento prematuro se asociaron con la HIM. Se ha documentado que el bajo peso al nacer se asocia con bajos niveles de calcio, fósforo y oxígeno, debido a que los pulmones aún no están totalmente desarrollados. La reducción en el abastecimiento de oxígeno para los ameloblastos durante la amelogénesis puede resultar en un esmalte defectuoso (17-22). Wu encontró en su estudio que los niños con bajo peso al nacer tienen tres veces más riesgo de presentar HIM $\left({ }^{19}\right)$.

Hipoxia: Se ha demostrado que la hipoxia perturba la amelogénesis en fetos de roedores. El episodio hipóxico puede dar lugar a defectos tanto cuantitativos como cualitativos en el esmalte, lo que refleja la vulnerabilidad de los ameloblastos. La ratas expuestas a hipoxia severa presentaban defectos del esmalte en forma 
de hipoplasia. $\left({ }^{3}\right)$ mientras que tanto la hipomineralización como la hipoplasia fueron observadas en ratones después de un ataque hipóxico agudo. $\left({ }^{24}\right)$.

Las disminuciones en el suministro de oxígeno inician mecanismos de adaptación diseñados para mantener la actividad celular a un nivel mínimo no obstante la falla de estos mecanismos durante la hipoxia produce disfunción celular y puede provocar daños celulares irreversibles $\left({ }^{25}\right)$.

Sin embargo, se especula que los mecanismos compensatorios como el aumento en la expresión del factor de inducible por la hipoxia cuya sigla en inglés es HIF-1 $\alpha$, se muestran regularmente altas en células ameloblastos después de la hipoxia, podría ser la razón para no desarrollar HIM en pacientes con hipoxia leve $\left({ }^{26}\right)$.

\section{POSNATALES}

Procesos febriles: La fiebre alta persistente influye en el proceso de formación del esmalte, ya que produce la desorientación de los prismas del esmalte en la etapa de formación y áreas sin cristales $\left({ }^{27,28}\right)$. Wakita et al. observaron que la fiebre causa alteración morfológica en el proceso de Tomes, lo cual provoca cambios en la forma de los prismas. Cabe resaltar que la fiebre causa defectos en el esmalte, mas no hay estudios que indiquen que causa la enfermedad $\left({ }^{29}\right)$.

Enfermedades como otitis media, neumonía, asma: Varios estudios relacionan el asma con el HIM y la explicación podría ser la siguiente: Estas condiciones que afectan el $\mathrm{pH}$ de la matriz del esmalte, es decir, la acidosis respiratoria y los niveles anormales de oxígeno resultantes de la hipoventilación en diversas enfermedades respiratorias inhiben la acción de las enzimas proteolíticas y el desarrollo de la hidroxiapatita cristalina que provoca la hipomineralización del esmalte $\left({ }^{30-32}\right)$.

Traumatismos: Farah et al. $\left({ }^{33}\right)$ concluyeron que un mayor contenido de albúmina y otras proteínas séricas como alfa-1-antitripsina y antitrombina III se encontraron dentro del esmalte afectado. Podría deberse a un trauma o injuria que genera una alteración en el poder de reabsorción de la matriz orgánica del esmalte y a la inhibición de las enzimas proteolíticas, lo que supondría la retención de proteínas e interferencia con la formación de cristales al faltar espacio para la deposición de los minerales causaría este defecto.

Hipomineralización de molar deciduo: El estudio de Tourino también encontró que un $38,7 \%$ de niños brasileros de su estudio que presentaba HIM había presentado hipomineralziación en las segundas molares deciduas ya que desarrollo de esta y del primer molar permanente comienza en el mismo momento, pero la fase de maduración del molar permanente es considerablemente más larga $\left({ }^{30}\right)$.

Uso de antibióticos: Wuollet et al. $\left({ }^{34}\right)$ observaron que la amoxicilina ocasionaba disturbios en el desarrollo del esmalte, pues su uso prolongado producía una baja organización en la formación del ameloblasto. Souza et al. encontraron que la amoxicilina interfiere en la fase inicial de la amilogénesis de los primeros molares, donde presenta un cambio en la estructura del ameloblasto y una reducción de la matriz del esmalte ${ }^{(35)}$. Además, la amoxicilina con el ácido clavulámico, consumidos por más de 60 días, afecta la función de los ameloblastos en su calidad y cantidad, especialmente en la fase de maduración36. También se observó que los macrólidos alteran el funcionamiento del ameloblasto en la etapa de transición $\left({ }^{37}\right)$. 
Uso de antiinflamatorios: Los antiinflamatorios son conocidos como inhibidores de la ciclooxigenasa, que son enzimas que catalizan la formación de postaglandinas del ácido araquidónico, mediante un proceso fisiológico severo, incluyendo la inflamación. Hay dos COX isoenzimas: COX 1, que es la enzima constitutiva, y COX 2, que es la enzima inductible que produce una respuesta rápida al aumento de los niveles intracelulares de óxido nítrico, citoquinas y calcio intracelular. Se refiere que la mineralización del esmalte requiere mediadores de la inflamación, debido al alto requerimiento de entrada de iones de los ameloblastos durante la formación de cristales. Por lo tanto, la participación de la COX 2 en la maduración del esmalte produce cambios en el contenido del calcio y fósforo $\left({ }^{38,39}\right)$. El estudio de Serna et al.40 mostró que la COX 2 está presente en el órgano del esmalte $y$, después de un tratamiento con antibióticos, acetaminofen e ibuprofeno, encontraron una disminución de la COX 2 y del contenido de calcio y fósforo en la matriz del esmalte. La rápida difusión de los nutrientes dentro de la capa del ameloblasto es necesaria durante el estadio de maduración, así como la incorporación rápida de los iones requeridos para el correcto crecimiento del cristal $\left({ }^{41}\right)$.

\section{CONCLUSIONES}

La causa exacta aún no está del todo definida, pero a partir de los estudios tenemos indicios que nos podrían ayudar a prevenir este tipo de defectos. Sin embargo, son necesarios más estudios longitudinales para determinar la etiología exacta de esta alteración en la formación dental para poder prevenirla, mientras que los médicos pediatras y odontopediatras deben estar conscientes de que el manejo temprano y adecuado de estas del HIM es importante.

Contribución del autor: María Eulalia Solís Espinoza, Catherine Sharlot Alarcón Calle han participado en la concepción del artículo, la recolección de información, su redacción y aprobación de la versión final.

Fuente de financiamiento: Autofinanciado.

Conflicto de interés: Las autoras declaran no tener conflicto de interés de ningún tipo. 


\section{REFERENCIAS BIBLIOGRÁFICAS}

1. Koch G, Hallonsten AL, Ludvigsson N, Hansson BO, Holst A, Ullbro C. Epidemiologic study of idiopathic enamel hypomineralization in permanent teeth of Swedish children. CommunityDent Oral Epidemiol. 1987; 15 (5): 279-85.

2. Weerheijm KL, Jalevik B, Alaluusua S. Molar-incisor hypomi-neralisation. Caries Res. 2001; 35 (5): 390-1.

3. Alaluusua S, Lukinmaa PL, Koskimies M, Pirinen S, Holtta P, Kallio M, Holtinnen T, Salmenpera L. Developmental dental defects associated with long breast feeding. Eur J Oral Sci. 1996; 104: 493-7.

4. Alaluusua S. Aetiology of molar-incisor hypomineralisation: A systematic review. Eur Arch Paediatr Dent. 2010; 11 (2): 53-8.

5. Weerheijm KL, Duggal M, Mejare I, Papagiannoulis L, Koch G, Martens LC, Hallonsten AL. Judgement criteria for molar incisor hypomineralisation (MIH) in epidemiologic studies: a summary of the European meeting on MIH held in Athens, 2003. Eur J Paediatr Dent. 2003; 4 (3): 110-3.

6. Fearne J, Anderson P, Davis GR. 3D X-ray microscopic study of the extent of variations in enamel density in first permanent molars with idiopathic enamel hypomineralisation. Br Dent J. 2004; 196 (10): 634-8.

7. Suckling GW. Developmental defects of enamel-historical and present-day perspectives of their pathogenesis. Adv Dent Res 1989; 3 (2): 87-94.

8. Koroyucu M, Ozel S, Tuna EB. Prevalence an etiology of molar-incisors hypomineralization (MIH) in the city of Istambul. J Dent Sci. 2018; 13 (4): 318-28.

9. Clarkson J. Review of terminology, classifications, and indices of developmental defects of enamel. Adv Dent Res 1989; 3: 104-9.

10. Schwendicke F, Elhennawy K, Reda S, Bekes K, Manton DJ, Krois J. Global burden of molar incisor hypomineralization. J. Dent. 2018, 68, 10-1.

11. Dantas-Neta NB, Moura LF, Cruz PF, et al. Impact of molar-incisor hypomineralization on oral healthrelated quality of life in schoolchildren. Braz Oral Res. 2016; 30 (1): e117.

12. Weerheijm KL. Molar incisor hypomineralization $(\mathrm{MIH})$ : clinical presentation, aetiology and management. Dent Update. 2004; 31: 9-12.

13. Fagrell TG, Lingström $P$, Olsson $S$, Steiniger F, Nóren JG. Bacterial invasion of dentinal tubules beneath appatently intact but hypomineralized enamel in molar teeth with molar incisor hypomineralization. Int J Paediatr Dent. 2008; 18: 333-340.

14. Jälevik, B, Klingberg, GA. (2002). Dental treatment, dental fear and behaviour management problems in children with severe enamel hypomineralization of their permanent first molars. International Journal of Paediatric Dentistry, 12 (1), 24-32.

15. Jiménez D, Guevara J, Zenteno E, Hernández JC. Alteration of the sialylation pattern of the murine tooth germ after ethanol exposure. Birth Defects Res A Clin Mol Teratol. 2005; 73: 980-8. 
16. Elfrink ME, et al. Pre- and postnatal determinants of deciduos molar hypomineralisation in 6 year old children. The deneration R study. PLoS One. 2014; 9 (7): e91057.

17. Ghanim A, Manton D, Bailey D, Mariño R, Morgan M. Risk factors in the occurrence of molar-incisor hypomineralization amongst a group of Iraqi children. International Journal of Paediatric Dentistry, 2012; 23 (3): 197-206.

18. Chaimay B, Woradet S. Does prenatal ultrasound exposure influence the development of children? Asia Pac J Public Health 2008; 20: 31-8.

19. Wu X, Wang J, Li YH, Yang ZY, Zhou Z. Association of molar incisor hypomineralization with premature birth or low birth weight: systematic review and metaanalysis. The Journal of Maternal-Fetal \& Neonatal Medicine.2018.

20. Roggini M, Pepino D, D’Avanzo M, Andreoli GM, Ceccanti S, Capocaccia P. Respiratory distress in newborn: evaluation of chest X-rays. Minerva Pediatr 2010; 62: 217-9.

21. Suckling G, Elliott DC, Thurley DC. The production of developmental defects of enamel in the incisor teeth of penned sheep resulting from induced parasitism. Arch Oral Biol 1983; 28: 393-9.

22. Chaimay B, Woradet S. Does prenatal ultrasound exposure influence the development of children? Asia Pac J Public Health 2008; 20: 31-8.

23. Shore RC, Whitford GM, Kirkham J, Brookes SJ, Robinson C. The effects of hypobaric hypoxia on rat incisor enamel organ. J Dent Res. 1998; 77: 642.

24. Sidaly R, Risnes S, Khan QE, Stiris T, Sehic A. The effect of hypoxia on the formation of mouse incisor enamel. Arch Oral Biol. 2015; 60 (11): 1601-12.

25. O'Rourke JF, Dachs GU, Gleadle JM, Maxwell PH, Pugh CW, Stratford IJ, Wood SM, Ratcliffe PJ. Hypoxia response elements. Oncol Res.1997; 9 (6-7): 327-32.

26. Sidaly R, Schmalfuss A, Sjaare AB, Sehic A, Stiris T, Espelid I. Five-minute Apgar score $\leq 5$ an Molar Incisor Hypomineralisation (HIM)- a case control study. BMC Oral Health. 2017; 17 (25): 1-7.

27. Tung $\mathrm{K}$, Fugita $\mathrm{H}$, Yamashita $\mathrm{Y}$, Sakagi $\mathrm{Y}$. Effect of turpenine- induced fever during the enamel formation of rat incisor. Arch Oral Biol. 2006; 51: 464-70.

28. Kuochung T, Haruko F, Yasuo Y, Yuzo T. Effect of turpentine-induced fever during the enamel formation of rat incisorArchives of Oral Biology. 2006;51: 464- 470.

29. Wakita M, Kobayashi S. The three dimensional structure of Tomes' processes and the development of the microsturctural organization of tooth enamel. In: Suga $\mathrm{S}$, editor. Mechanisms of tooth enamel formation. Tokyo: Quintessence; 1983. p. 65-89.

30. Tourino LFPG, Corrêa-Faria P, Ferreira RC, Bendo CB, Zarzar PM, Vale MP Association between molar incisor hypomineralization in schoolchildren and both prenatal and postnatal factors: a population-based study. PLoS ONE. 2016; 11 (6): e0156332. 
31. Garg N, Jain AK, Saha S, Singh J. Essentiality of early diagnosis of molar incisor hypomineralization in children and review of its clinical presentation, etiology and management. Int J Clin Pediatr Dent. 2012;5(3):190-196.

32. Kühnisch J, et al. Respiratory diseases are associated with molar-incisor hypomineralizations. Swiss Dental Journal. 2014; 124: 286-93.

33. Farah R, Monk BC, Swain MV, Drummond BK. Protein content of molar-incisor hypomineralization enamel. Journal of Dentistry. 2010; 38: 591-6.

34. Wuollet E, Laisi S, Salmela E, Ess A, Alaluusua S. Molar-incisor hypomineralization and the association with childhood illnesses and antibiotics in a group of Finnish children. Acta Odontol Scand. 2016; 74 (5): 416-22.

35. De Souza JF, Gramasco M, Jeremias F, et al. Amoxicillin diminishes the thickness of the enamel matrix that is deposited during the secretory stage in rats. Int $\mathrm{J}$ Paediatr Dent. 2016; 26: 199-210.

36. Mihalas E, Matricala L, Chelmus A, et al. The role of chronic exposure to amoxicillin/clavulanic acid on the developmental enamel defects in mice. Toxicol Pathol. 2016; 44: 61-70.

37. Abe T. Effects of a macrolide antibiotic on enamel formation in rat incisors-primary lesion of ameloblast at the transition stage. J Vet Med Sci. 2003; 65: 985-8.

38. Flower R. The development of COX 2 inhibitors. Nat Rev Drug Discov 2003;2(3):179-91

39. Rainsford K. Ibuprofen: pharmacology, efficacy and safety. Inflammopharmacology. 2009; 17 (6): 275-342.

40. Serna C, Pérez A, Solano F, Castells M, Vicente A, Ortiz A. Effect of antibiotics and NSAIDs on cyclooxygenase-2 in the enamel mineralization. Sci Rep. 2018; 8 (1): 4132.

41. Takano Y. Enamel mineralization and the role of ameloblasts in calcium transport. Connect Tissue Res. 1995; 33 (1-3): 127-37. 